\title{
Macular Function after PDT in Myopic Maculopathy: Psychophysical and Electrophysiological Evaluation
}

\author{
Monica Varano, ${ }^{1}$ Vincenzo Parisi, ${ }^{1}$ Massimiliano Tedeschi, ${ }^{1}$ Marta Sciamanna, ${ }^{1}$ \\ Geltrude Gallinaro, ${ }^{1}$ Nicoletta Capaldo, ${ }^{1}$ Susanna Catalano, ${ }^{2}$ and Antonella Pascarella ${ }^{2}$
}

Purpose. To evaluate the effects of photodynamic therapy (PDT) on macular function in myopic subfoveal choroidal neovascularization (CNV).

Methods. Fourteen eyes of 14 patients (mean age, $48.1 \pm 13.3$ years) with myopic CNV (myopia ranging from -6.50 to -20 D) were enrolled. In each eye, at baseline and at 15 and 90 days after PDT with verteporfin, logMAR visual acuity (logarithm of the minimum angle of resolution VA), macular sensitivity by scanning laser ophthalmoscope microperimetry, and focal $\left(\right.$ central $\left.9 \times 9^{\circ}\right)$ ERGs (FERGs) and pattern ERGs (PERGs) were assessed.

Results. At 15 days after PDT, myopic CNV eyes showed, in relation to baseline values, a significant (ANOVA, $P<0.01$ ) reduction in the diameter of the lesion that correlated (Pearson test, $P<0.01$ ) with the significant (ANOVA, $P<0.01$ ) increase in FERG and PERG amplitudes, VA, and scanning laser ophthalmoscopy (SLO) microperimetry results obtained from the central $1^{\circ}$ to $2^{\circ}$ of the macular area (SLO-CM). At 90 days after PDT, myopic CNV eyes showed, in comparison with baseline values, a nonsignificant (ANOVA, $P>0.01$ ) reduction in the diameter of the lesion, a nonsignificant increase in VA and SLO-CM, and a still significant increase in FERG and PERG amplitudes.

Conclusions. In myopic CNV eyes, PDT induces an increase, though not significant, in VA and macular sensitivity. These changes may be related to a reduction in the diameter of the lesion, with an improvement in the function of both ganglionic and preganglionic elements of the macular region, as suggested by the improvement in FERG and PERG responses. (Invest Ophthalmol Vis Sci. 2005;46:1453-1462) DOI:10.1167/iovs.04-0903

$\mathrm{P}$ athologic myopia of at least $-6 \mathrm{D}$ has been reported to be the seventh leading cause of legal blindness in the United States. $^{1-3}$ One of the most prevalent complications of the disease is macular choroidal neovascularization (CNV; juxta- or subfoveal), which occurs in $5 \%$ to $10 \%$ of myopic eyes with a refraction of $-6 \mathrm{D}^{4}$ and which causes a substantial risk of severe visual loss, reported to be between $43 \%$ and $51 \%$ and $58 \%$ and $76 \%$ at $2^{5,6}$ and 5 to 10 years, ${ }^{3,7}$ respectively, after development of $\mathrm{CNV}$.

In such cases of myopic $\mathrm{CNV}$, laser photocoagulation may provide a short-term benefit, but several studies have reported that after a few years, there is no difference, in comparison to

From the ${ }^{1}$ Fondazione per l'Oftalmologia G. B. Bietti, Roma, Italy; and ${ }^{2}$ Ospedale Oftalmico, Roma, Italy.

Submitted for publication July 29, 2004; revised November 29, 2004; accepted January 2, 2005.

Disclosure: M. Varano, None; V. Parisi, None; M. Tedeschi, None; M. Sciamanna, None; G. Gallinaro, None; N. Capaldo, None; S. Catalano, None; A. Pascarella, None

The publication costs of this article were defrayed in part by page charge payment. This article must therefore be marked "advertisement" in accordance with 18 U.S.C. $\$ 1734$ solely to indicate this fact.

Corresponding author: Vincenzo Parisi, Fondazione per l'Oftalmologia G.B. Bietti, Via Livenza 3, 00198, Roma, Italy; vparisi@tin.it. untreated eyes. ${ }^{3,7}$ Photocoagulation is also complicated by a high rate of recurrences and by subfoveal extension of the laser scar. ${ }^{7,8}$ Moreover, thermal destruction can rarely be applied because approximately one half to three fourths of the CNVs are already subfoveal at initial presentation. ${ }^{5,7}$ In addition, conflicting results have been reported with submacular surgery in these patients. 9,10

Recently, photodynamic therapy (PDT) with verteporfin has been introduced into clinical practice as a new therapeutic modality for treating subfoveal $\mathrm{CNV}$ in patients affected by age-related macular degeneration (AMD) $)^{11-13}$ as well as in eyes with myopic CNV. ${ }^{14-16}$ Indeed, PDT for myopic CNV has been reported to reduce substantially the rate of visual loss after 1 year. ${ }^{14-17}$

Macular function can be evaluated by psychophysical or electrophysiological methods. Scanning laser ophthalmoscope (SLO) microperimetry represents a psychophysical method that allows performance of manual static perimetry, to seek the retinal sensitivity thresholds of 30 to 40 points in the macular area, under ophthalmoscopic monitoring. ${ }^{18-20}$ An electrophysiological evaluation of macular function can be obtained by recording ERG signals in response to luminance (focal ERG [FERG]) or contrast-modulated (pattern ERG [PERG]) stimuli presented in the central $9^{\circ} .{ }^{21}$ FERG allows an electrophysiological evaluation of the function of the outer retinal layers (preganglionic elements ${ }^{21-27}$ ) of the macular region, whereas PERG allows an electrophysiological evaluation of the function of the innermost retinal layers (ganglion cells and their fibers $\left.^{21,27-29}\right)$ of the macular region.

In our study, we sought to evaluate the effects of PDT on macular function in myopic CNV by psychophysical methods (visual acuity [VA], SLO microperimetry), and the related changes in function of both the outer and innermost retinal macular layers, by evaluating FERG and PERG responses.

\section{Methods}

\section{Patients}

Sixty-two patients (32 men and 28 women; mean age, $51.2 \pm 14.4$ years) with subfoveal $\mathrm{CNV}$ due to pathologic myopia (ranging from -6.50 to $-20 \mathrm{D}$ ) were examined for enrollment in the study.

Because several diseases may influence SLO microperimetry, VA, and FERG and PERG responses, ${ }^{21}$ we excluded patients with the presence of moderate to dense lens opacities $(n=6)$, implanted intraocular lenses $(n=3)$, corneal opacities $(n=1)$, a history of refractive surgery $(n=5)$, glaucoma or ocular hypertension $(n=4)$, a history of intraocular inflammation such as anterior or posterior uveitis $(n=1)$, multifocal choroiditis $(n=4)$, a history of retinal detachment $(n=3)$ or laser treatment for peripheral retinal diseases ( $n$ $=7)$, diabetes $(n=3)$, systemic hypertension in medical treatment $(n$ $=5)$, connective tissue disease $(n=1)$, a history of ocular trauma ( $n$ $=1$ ), optic neuropathy $(n=1)$, or other systemic or neurologic diseases $(n=3)$.

Therefore, 14 eyes of 14 patients ( 8 men, 6 women; mean age, $48.1 \pm 13.3$ years) with subfoveal $\mathrm{CNV}$ due to pathologic myopia (range, -6.50 to $-20 \mathrm{D}$ ) were enrolled in the study (14 myopic CNV 
eyes). The fellow eyes of the 14 enrolled patients were emmetropic ( 1 eye) and myopic (ranging from -6.50 to $-20 \mathrm{D}$ ) but without CNV or other macular diseases (13 eyes).

The clinical diagnosis of CNV was based on fluorescein angiography. At baseline conditions, the fluoroangiographic criterion was evidence of fluorescein leakage from $\mathrm{CNV}$ caused by pathologic myopia, extending under the geometric center of the avascular zone. The lesion could include other features obscuring $\mathrm{CNV}$ identification by fluorescein angiography, including blood, hypofluorescence not arising from visible blood, or serous detachment of the retinal pigment epithelium (RPE). During fluorescein angiography, the greatest linear dimension of the lesion was measured (Imagenet System; Topcon, Tokyo, Japan). Hemorrhages were considered an integral part of the lesion, in agreement with the VIP protocol. ${ }^{14}$

Myopic CNV eyes were compared with 16 eyes of 16 age-matched control subjects (16 control eyes [CEs]). The same exclusion criteria used for myopic patients were applied to control subjects. In CEs, the refractive error was \pm 0.50 spherical diopters.

VA, macular sensitivity by SLO microperimetry, FERGs, and PERGs were assessed at a baseline examination in all subjects and patients, using the following methods.

\section{Best Corrected VA}

Best corrected VA was assessed by the modified ETDRS chart (Lighthouse Low Vision Products, Long Island City, NY). VA was expressed as logMAR obtained at distances of $4,2,1$, and $0.5 \mathrm{~m}$.

\section{SLO Microperimetry}

Macular sensitivity was evaluated by SLO microperimetry (Rodenstock, Dusseldorf, Germany). SLO microperimetry permits an exact, point-topoint correspondence between fundus image and perimetric results. A helium-neon laser beam $(633 \mathrm{~nm})$ and an infrared diode laser $(780 \mathrm{~nm})$ are simultaneously projected onto the retina and detected through a selection of confocal apertures. The infrared laser, which is invisible to the patient, is used for retinal imaging, allowing a satisfactory exploration when lens or vitreous opacities are present. Retinal illumination for psychophysical testing is obtained by a HeNe laser beam. This laser, used for the generation of the background and stimulus illumination, is modulated via an acousto-optic modulator. ${ }^{18-20}$ Confocal SLO with graphic capabilities allows the investigator to determine the retinal location of visual stimuli directly on the retinal image in real time. The stimuli are observed by the patient while the images of the stimuli are directly seen on the patient's retina by the observer.

The central fixation stimulus is a cross directly projected onto the foveal area, visualized on the monitor display. The search for liminal values by static manual perimetry is performed by testing 25 to 30 points in the macular area, starting from a supraliminal stimulus. The area of perimetric examination comprises either $33 \times 21^{\circ}$ or $16.5 \times$ $10.5^{\circ}$. We prefer the first image consisting of $512 \times 512$ pixels. SLO provides a $33 \times 21^{\circ}$ image of the fundus with a minimum resolution of 4 minutes of arc $(20 \mu \mathrm{m})$ for measurement and positioning of targets. Microperimetry can be performed with a different-sized point stimulus, ranging from Goldmann I to IV. ${ }^{18-20}$ A stimulus size equivalent to the Goldmann II test spot was used. The intensity of the stimulus is inversely related to the tested retinal sensitivity, expressed as alphabetic letters from $\mathrm{A}$ (corresponding to $0 \mathrm{~dB}$ ) to $\mathrm{g}$ (corresponding to 32 $\mathrm{dB})$. Missed stimuli, represented by the corresponding letter, are also stored and visualized with a small, dark square.

Manual static perimetry was performed with a concentric grid centered on the fovea and rested on the monitor of the SLO. The first stimulus was presented at the level of $15 \mathrm{~dB}$. Thereafter, light intensity was increased by $2 \mathrm{~dB}$ after a correct answer and decreased by $1 \mathrm{~dB}$ if the stimulus was not perceived. The operator selected a retinal landmark, such as a vessel bifurcation, that could be easily identified. Immediately after the detection of each single stimulus, the operator positioned a cursor over the retinal landmark, which enabled the computer to calculate the real point of fixation as well as the actual location of the stimulus.

We studied macular sensitivity, testing the mean values, expressed in decibels, of eight retinal points (two points for each quadrant: superior, nasal, inferior, and temporal) of the central $1^{\circ}$ to $2^{\circ}$ of the macula (central microperimetry, SLO-CM) and the mean value at 16 to 20 retinal points ( $4-5$ points in each quadrant) dislocated in an annular area from the central $2^{\circ}$ to $7^{\circ}$ (paracentral microperimetry, SLO-PM). In addition, in eyes with CNV, SLO examination allowed us to assess the relationship between the retinal fixation locus and CNV. Thus, the location of the retinal fixation locus in relation to CNV was assessed in each patient by considering the macular region as divided into four parts (superior, inferior, nasal, and temporal). When the macular area was sufficiently tested, the digital fundus image was frozen and saved on the hard disc.

At baseline, in each patient or control subject, SLO microperimetry was performed three times on three different days. The last evaluation was considered in the statistical analysis and the test-retest variability was evaluated on the basis of the second and third evaluations.

In the subsequent SLO assessments, performed at 15 and 90 days after PDT, we repeated the procedure, choosing the same concentric grid centered on the functional fixation point and selecting the same retinal landmark as in the baseline evaluation. In subsequent SLO assessments, performed at 15 and 90 days after PDT, the same procedure was repeated. The same number of tested points was evaluated for CM and PM (two points for each quadrant for SLO-CM and four to five points for each quadrant for SLO-PM.). In four patients, the number of tested points at the repeat examinations was greater than that recorded at baseline. These additional perimetric locations were excluded from further analysis.

\section{FERG and PERG}

FERGs were recorded in response to a $9 \times 9^{\circ}$ uniform field, flickered sinusoidally at $30 \mathrm{~Hz}$ (mean luminance, $80 \mathrm{~cd} / \mathrm{m}^{2}$; modulation depth, 93.5\%). ${ }^{26}$ PERGs were recorded in response to sinusoidal gratings of 2 cyc/deg spatial frequency, $90 \%$ contrast, generated in the TV monitor (mean luminance, $80 \mathrm{~cd} / \mathrm{m}^{2}$ ) and modulated in counterphase at $8 \mathrm{~Hz}$, stimulating the central $9 \times 9^{\circ}{ }^{28,29}$ To minimize the stray light effect in both FERG and PERG recordings, an equiluminous large surround $\left(120 \times 120^{\circ}\right)$ was peripherally provided.

Both FERG and PERG recordings were performed with full correction of refraction at the viewing distance. A small, red fixation target, subtending a visual angle of approximately $0.5^{\circ}$ (estimated after taking into account spectacle-corrected individual refractive errors) was placed at the center of the pattern stimulus. At every ERG examination, each patient positively reported that he or she could clearly perceive the fixation target. Pupils were pharmacologically dilated to 8 to $9 \mathrm{~mm}$. Stimulation was monocular after occlusion of the other eye.

The bioelectrical signal was recorded by a small $\mathrm{Ag} / \mathrm{AgCl}$ skin electrode placed over the lower eyelid. FERGs and PERGs were derived bipolarly between the stimulated (active electrode) and the patched (reference electrode) eye, by a previously described method. ${ }^{30}$ The ground electrode was placed at Fpz. Interelectrode resistance was $<3$ $\mathrm{K} \Omega$.

The signal was amplified (gain 50,000), filtered (band-pass, 1-30 $\mathrm{Hz}$ ), and averaged with automatic rejection of artifacts (200 events free of artifact were averaged for every trial; model BM 6000; Biomedica Mangoni, Pisa, Italy). Analysis time was $33.2 \mathrm{~ms}$ in FERG recordings and $125 \mathrm{~ms}$ in PERG recordings. At least three consecutive FERGs and PERGs were recorded and the waveform stored on the computer screen to evaluate intraindividual variability of the bioelectrical responses.

Acquired signals underwent Fourier analysis to isolate the fundamental harmonic component (FERG: temporal frequency of $30 \mathrm{~Hz}$, first harmonic: 1F; PERG: temporal frequency of $8 \mathrm{~Hz}$, second harmonic: $2 \mathrm{P}$ ), whose amplitude (in microvolts) and phase (in degrees) were measured. We accepted FERG and PERG signals with a signal-to-noise 
ratio $>2$. The noise was measured by recording the bioelectrical signals (200 averaged events), while the monitor was screened by a cardboard, and noise $<0.1 \mu \mathrm{V}$ (mean $0.082 \mu \mathrm{V}$ ) was observed in all subjects tested. At baseline, FERG and PERG recordings were repeated on two separate days in both control subjects and patients, to assess test-retest variability.

\section{Photodynamic Therapy}

In patients affected by myopic CNV, PDT was carried out as recommended by the VIP study. ${ }^{14}$ Briefly, a dose of verteporfin (Visudyne; Novartis, Basel, Switzerland) of $6 \mathrm{mg} / \mathrm{m}^{2}$ of body surface area was administered via intravenous infusion of $30 \mathrm{~mL}$ over 10 minutes. Fifteen minutes after the onset of verteporfin infusion, a 689-nm laser light was delivered at an intensity of $600 \mathrm{~mW} / \mathrm{cm}^{2}$ over 83 seconds to provide a light dose of $50 \mathrm{~J} / \mathrm{cm}^{2}$ to a round spot on the retina. The spot's diameter was calculated to be $1000 \mu \mathrm{m}$ larger than the greatest linear dimension of the $\mathrm{CNV}$ lesion.

\section{Follow-up}

Fluorescein angiography with relative measurement of the greatest linear diameter of the lesion, VA, SLO microperimetry and FERG and PERG recordings were assessed in myopic CNV eyes 15 and 90 days after PDT.

The research followed the tenets of the Declaration of Helsinki, and the study was approved by the local ethics committee

\section{Statistics}

Results from CE and myopic CNV eyes observed at baseline conditions were compared by one-way analysis of variance (ANOVA). Changes in functional (VA, SLO-CM, SLO-PM, FERG, and PERG) and morphometric (greatest linear diameter of the lesion) parameters observed in $\mathrm{CNV}$ eyes 15 and 90 days after PDT were compared with baseline (pretreatment) values by one-way ANOVA

The Pearson correlation was used to correlate the diameter of the lesion with all parameters of visual function (logMAR VA, SLO-CM and -PM sensitivities, and PERG 2P and FERG 1F log amplitudes) and to correlate logMAR VA values with the other parameters of visual function (SLO-CM and -PM sensitivities and PERG 2P and FERG 1F log amplitudes). FERG and PERG amplitudes and morphometric data from the lesion (greatest linear dimension) underwent logarithmic transformation for better approximation of a normal distribution. Test-retest data for SLO microperimetry and FERG and PERG results are expressed as the mean difference between two records obtained in separate sessions \pm the standard deviation of the difference. The $95 \%$ confidence limits of test-retest variability in normal subjects and patients were established assuming a normal distribution.

In all analyses, $P<0.01$ was considered statistically significant.

\section{Results}

\section{Baseline Examination and Measurements}

The clinical characteristics of CNV eyes observed at baseline are reported in Table 1 (baseline). Figure 1B shows an example of fluorescein angiography in one CNV eye (CNV 1). Figure 2B shows an example of SLO microperimetry in one CNV eye (CNV 8). PERG and FERG recordings assessed in the same eye $(\mathrm{CNV} 8)$ are shown in Figure 3. Mean data are presented in Table 2 .

Test-retest data for SLO microperimetry were as follows: control subjects, mean $0.48 \mathrm{~dB}$, SD $1.23 \mathrm{~dB} ; 95 \%$ confidence limits $-1.98,2.94 \mathrm{~dB}$; CNV eyes, mean $0.52 \mathrm{~dB}$, SD $1.79 \mathrm{~dB}$; 95\% confidence limits $-3.06,4.10 \mathrm{~dB}$.

Test-retest data for PERG amplitudes were as follows: control subjects, mean $0.0006 \mathrm{~dB}(0.0043 \mu \mathrm{V})$, SD $0.030 \mathrm{~dB}(0.106$ $\mu \mathrm{V}) ; 95 \%$ confidence limits $-0.0594,0.6006 \mathrm{~dB}(-0.207$, $0.216 \mu \mathrm{V})$; CNV eyes, mean $0.015 \mathrm{~dB}(0.016 \mu \mathrm{V}), \mathrm{SD} 0.0727 \mathrm{~dB}$

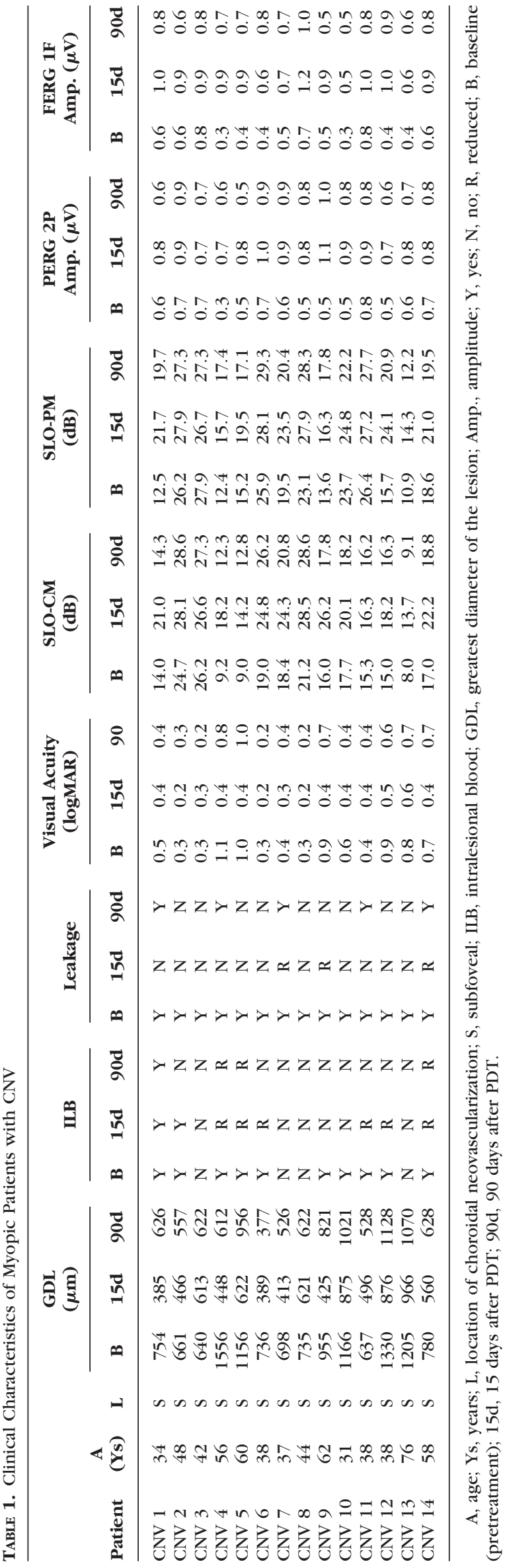



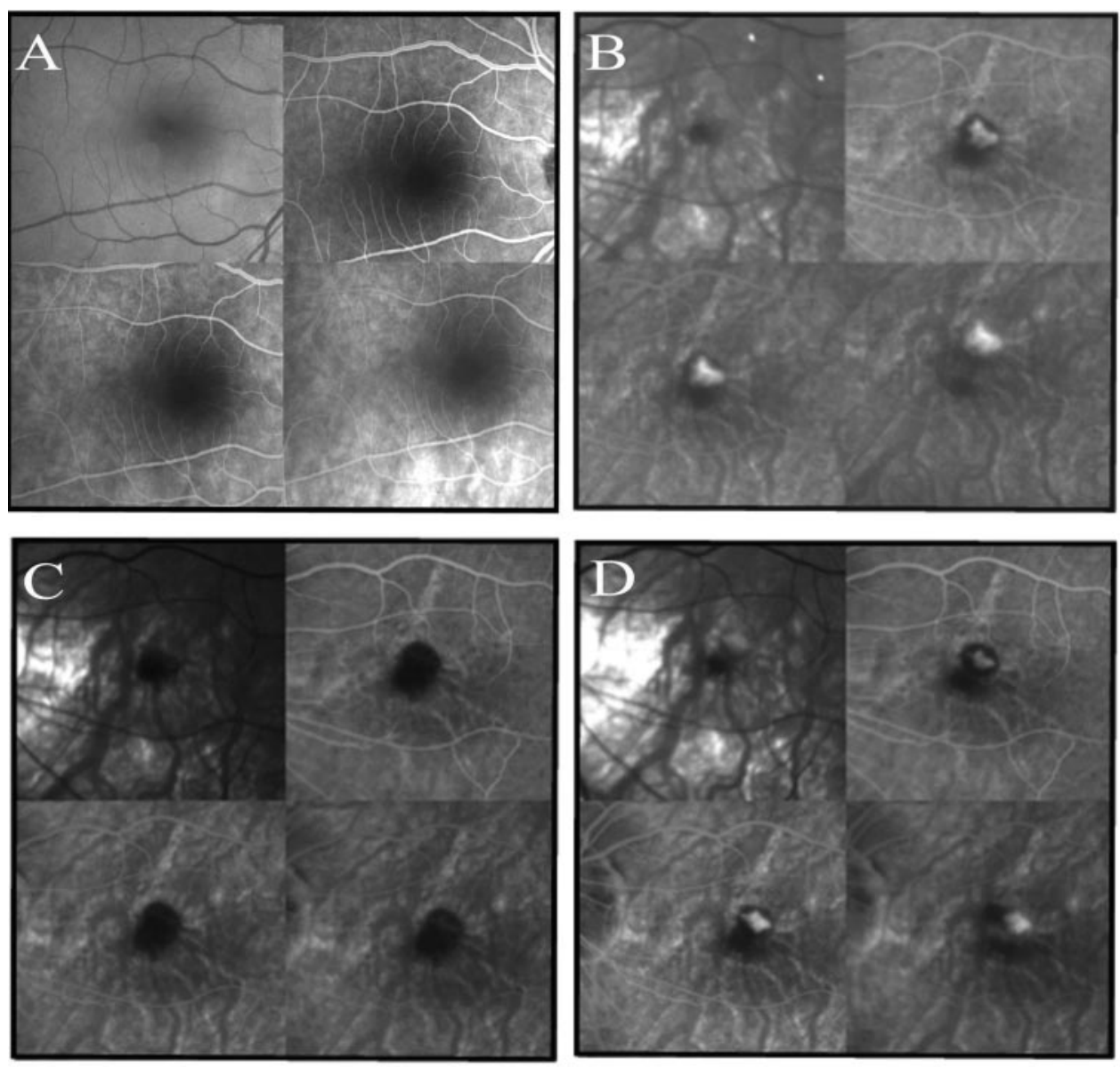

Figure 1. Examples of fluorescein angiographs of one control eye (A) and one myopic $\mathrm{CNV}$ eye (CNV 1) at baseline (B) and at 15 (C) and 90 (D) days after PDT. Before PDT, a CNV lesion with evident leakage was present in the macular area of the myopic $\mathrm{CNV}$ eye. At 15 days after PDT, a significant reduction of the diameter of the lesion and absence of leakage was observed. At 90 days after PDT, the myopic CNV eye showed an increase in the diameter of the lesion and the reappearance of leakage.

$(0.099 \mu \mathrm{V}), 95 \%$ confidence limits $-0.129,0.213 \mathrm{~dB}(-0.182$, $0.214 \mu \mathrm{V})$.

Test-retest data for FERG amplitudes were as follows: control subjects, mean $0.0029 \mathrm{~dB}(0.007 \mu \mathrm{V})$, SD $0.028 \mathrm{~dB}(0.097$ $\mu \mathrm{V}) ; 95 \%$ confidence limits $-0.0531,0.0589 \mathrm{~dB}(-0.187$, $0.201 \mu \mathrm{V})$; CNV eyes, mean $0.011 \mathrm{~dB}(0.007 \mu \mathrm{V})$, SD $0.079 \mathrm{~dB}$ $(0.094 \mu \mathrm{V}) ; 95 \%$ confidence limits $-0.147,0.169 \mathrm{~dB}(-0.181$, $0.195 \mu \mathrm{V})$.

CNV eyes showed a diameter of the lesion ranging from 637 to $1556 \mu \mathrm{m}$. Intralesional blood was present in $10 \mathrm{CNV}$ eyes and absent in 4, and leakage was present in all.

CNV eyes showed logMAR VA, SLO-CM sensitivity, and PERG $2 \mathrm{P}$ and FERG $1 \mathrm{~F}$ amplitudes significantly $(P<0.01)$ reduced and PERG $2 \mathrm{P}$ and FERG $1 \mathrm{~F}$ phases significantly delayed $(P<0.01)$ when compared with CEs. SLO-PM in CNV eyes was reduced, although not significantly $(P=0.092)$, compared with those of CEs.

In $\mathrm{CNV}$ eyes, the retinal fixation locus was, in relation to the $\mathrm{CNV}$, superior in four right eyes (CNV 2, CNV 3, CNV 7, and CNV 11), superonasal in four left eyes (CNV 4, CNV 6, CNV 9, and CNV 12), superotemporal in two right eyes (CNV 5 and CNV 14), inferonasal in one left eye (CNV 1), inferior in one left eye (CNV 10), temporal in one left eye (CNV 13), and nasal in one left eye (CNV 8).

Table 3 reports the correlation between $\log$ MAR VA, SLO-CM and -PM sensitivities, and PERG 2P and FERG 1F amplitudes of individual $\mathrm{CNV}$ eyes and the corresponding diameter of the lesion. Significant correlations $(P<0.01)$ were observed between the diameter of the lesion and parameters of visual function.

Table 3 also reports the correlation between logMAR VAs of individual CNV eyes and the corresponding SLO-CM and -PM sensitivities, and PERG 2P and FERG 1F amplitudes. Significant correlations $(P<0.01)$ were observed between logMAR VA and the other parameters of visual function.

\section{Fifteen Days after PDT}

The clinical characteristics of CNV eyes observed 15 days after PDT are reported in Table 1 (15 days). An example of fluorescein angiography in one CNV eye (CNV 1) is shown in Figure 1C. Figure $2 \mathrm{C}$ shows an example of SLO microperimetry in one CNV eye (CNV 8). PERG and FERG recordings, assessed in the same eye ( $\mathrm{CNV} 8)$, are shown in Figure 3. Mean data are presented in Table 2 .

Fifteen days after PDT, CNV eyes showed a significant $(P<$ $0.01)$ reduction of the diameter of the lesion. Intralesional blood was still present in two CNV eyes, reduced in six, and absent in six. (Of the latter, at baseline two had intralesional blood, whereas in four it was absent.) Leakage was absent in 11 $\mathrm{CNV}$ eyes and reduced in 3 . There was a significant $(P<0.01)$ increase in logMAR VA, SLO-CM sensitivity, and PERG $2 \mathrm{P}$ and FERG $1 \mathrm{~F}$ amplitudes and a significant $(P<0.01)$ shortening in PERG $2 \mathrm{P}$ and FERG $1 \mathrm{~F}$ phases in relation to baseline in CNV eyes. We also observed an increase, though not significant $(P=$ 0.112 ), in SLO-PM sensitivity.

In Figure 4, on the left, the differences in logMAR VA, SLO-CM sensitivity, and PERG $2 \mathrm{P}$ and FERG $1 \mathrm{~F}$ amplitudes observed in individual $\mathrm{CNV}$ eyes in relation to baseline are plotted as a function of the corresponding difference in the diameter of the lesion. Significant correlations $(P<0.01)$ were observed between the reduction of the diameter of the lesion and the increase in logMAR VA, SLO-CM sensitivity, and PERG $2 \mathrm{P}$ and FERG $1 \mathrm{~F}$ amplitudes. No correlation $(P=0.300)$ was 

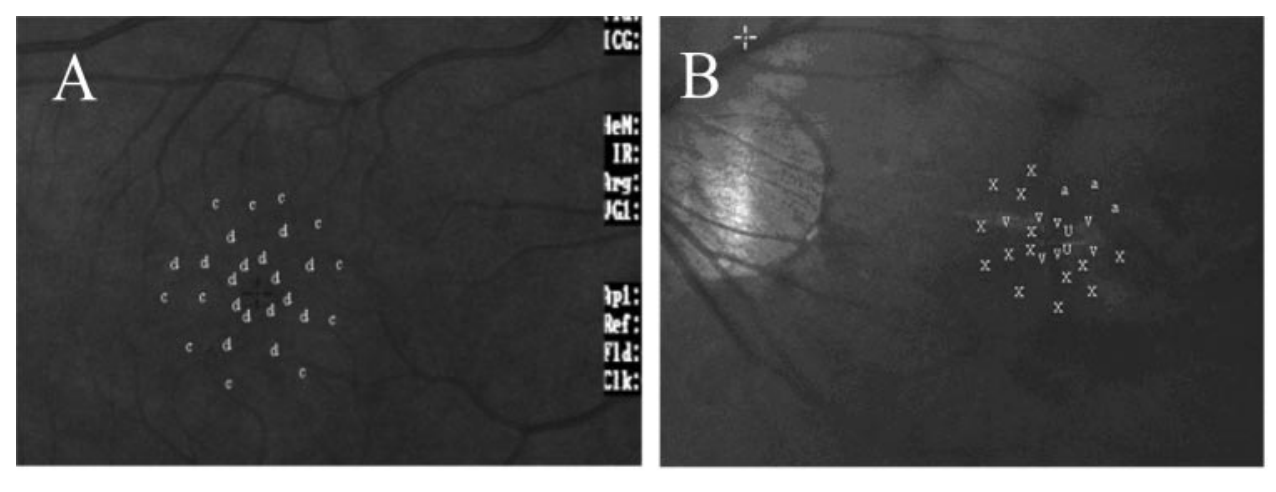

FiguRE 2. Example of SLO microperimetry in one control eye (A) and in one myopic $\mathrm{CNV}$ eye (CNV 8) at baseline (B) and at 15 (C) and 90 (D) days after PDT. Macular sensitivity was examined, by testing the mean decibels at eight retinal points of the central $1^{\circ}$ to $2^{\circ}$ of the macula (SLO$\mathrm{CM})$ and the mean decibels at 20 retinal points located in an annular area from the central $2^{\circ}$ to $7^{\circ}$ (SLOPM). The intensity of the stimulus inversely corresponded to the tested retinal sensitivity, expressed as an alphabetic representation of letters from A (corresponding to $0 \mathrm{~dB}$ ) to $\mathrm{g}$ (corresponding to $32 \mathrm{~dB}$ ). At baseline, CNV 8 showed a reduction in macular sensitivity in both SLO-CM and $-\mathrm{PM}$ in relation to the control eye. At 15 days after PDT, CNV 8 showed an increase in SLO-CM and -PM sensitivities. At 90 days after PDT, CNV 8 showed SLO-PM and -CM sensitivities similar to those observed 15 days after PDT.
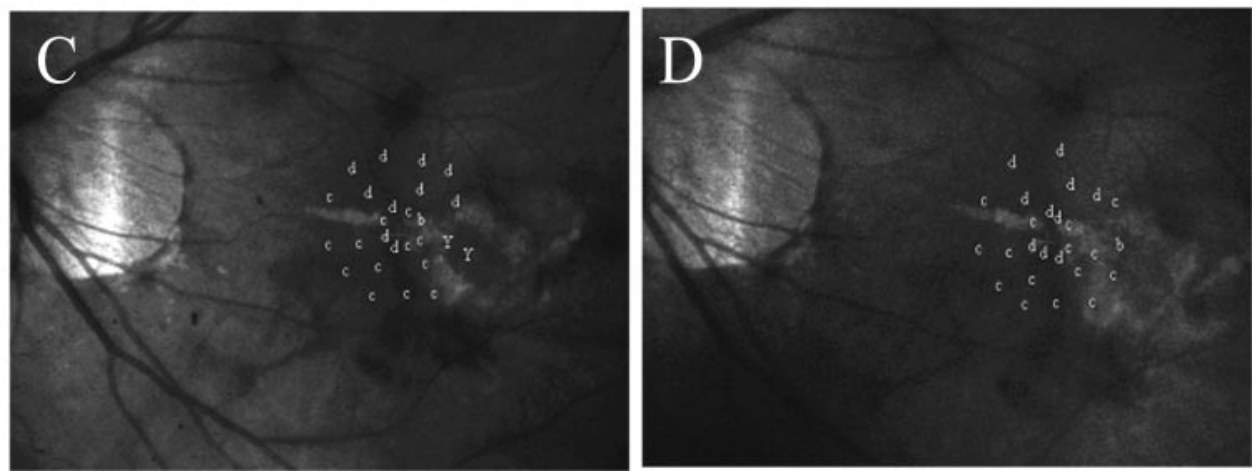

found between the reduction of the diameter of the lesion and changes in SLO-PM sensitivity.

In Figure 4, right, the differences in logMAR VA observed in individual $\mathrm{CNV}$ eyes in relation to baseline are plotted as a function of the corresponding differences in SLO-PM and -CM sensitivities and PERG $2 \mathrm{P}$ and FERG $1 \mathrm{~F}$ amplitudes. Significant correlations $(P<0.01)$ were observed between the increase in $\log$ MAR VA and SLO-CM sensitivity and PERG $2 \mathrm{P}$ and FERG $1 \mathrm{~F}$ amplitudes. No correlation was found $(P=0.475)$ between the increase in logMAR VA and changes in SLO-PM sensitivity.

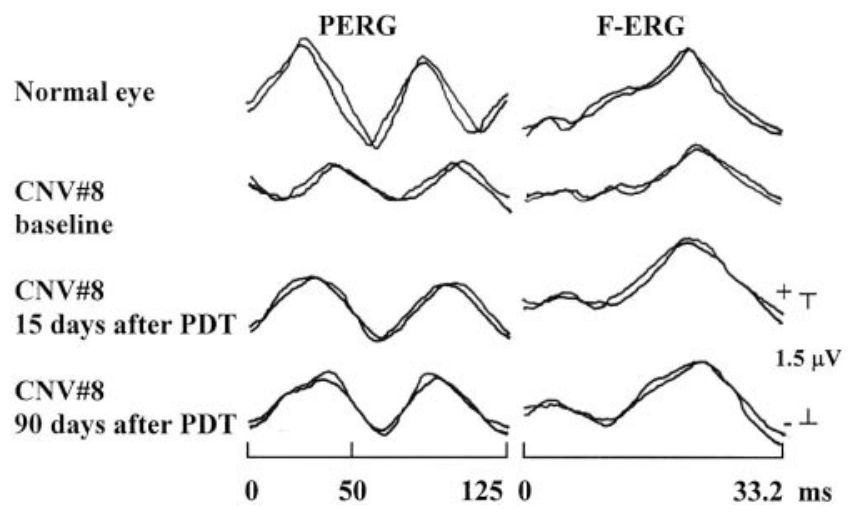

FiguRE 3. Examples of FERG and PERG recordings from one control eye and one myopic CNV eye (CNV 8) at baseline and 15 and 90 days after PDT. At baseline, CNV 8 showed FERG and PERG responses with reduced amplitudes and delayed phases in relation to the control eye. Fifteen days after PDT, CNV 8 showed increased amplitudes and shortened phases of FERG and PERG responses in relation to baseline recordings. Ninety days after PDT, PERG amplitudes and phases were still respectively increased and shortened in relation to baseline recordings and unmodified when compared to 15 days after PDT. FERG amplitude and phase of CNV 8 were respectively reduced and delayed compared with results at 15 days, but still improved when compared with baseline.

\section{Ninety Days after PDT}

The clinical characteristics of $\mathrm{CNV}$ eyes observed 90 days after PDT are reported in Table 1 (90 days). An example of fluorescein angiography in one CNV eye (CNV 1) is shown in Figure 1D. An example of SLO microperimetry in one CNV eye (CNV 8) is presented in Figure 2D. PERG and FERG recordings, assessed in the same eye (CNV 8), are shown in Figure 3. Mean data are presented in Table 2.

Ninety days after PDT, CNV eyes showed an increase in the diameter of the lesion with respect to the size observed 15 days after PDT, but the diameter was still reduced, though not significantly $(P=0.050)$, when compared with baseline. Intralesional blood was not present in the two CNV eyes with the presence of blood at 15 days and was reduced in three CNV eyes and absent in six CNV eyes. (In four of these, there was no intralesional blood at baseline.) Leakage was absent in nine $\mathrm{CNV}$ eyes and present in five.

A decrease in logMAR VA, SLO-CM and -PM sensitivities, and PERG 2P and FERG $1 \mathrm{~F}$ amplitudes and a delay in PERG $2 \mathrm{P}$ and FERG $1 \mathrm{~F}$ phases was found in relation to the values observed 15 days after PDT. Nevertheless, logMAR VA and SLO-CM and -PM sensitivities were still increased, though not significantly ( $P=0.305, P=0.254, P=0.253$, respectively), when compared with the baseline, PERG $2 \mathrm{P}$ and FERG $1 \mathrm{~F}$ amplitudes and PERG $2 \mathrm{P}$ and FERG $1 \mathrm{~F}$ phases were still significantly $(P<0.01)$ increased and shortened in relation to the values observed at baseline conditions.

The difference in the diameter of the lesion in relation to the baseline diameter did not correlate significantly $(P>0.01)$ with the corresponding difference in logMAR VA, SLO-CM and -PM sensitivities, and PERG 2P and FERG 1F amplitudes (Fig. 5, left). No significant correlations $(P>0.01)$ were observed between the difference in logMAR VA in relation to baseline and the corresponding difference in SLO-CM and -PM sensitivities and PERG 2P and FERG 1F amplitudes (Fig. 5, right). 
TABLE 2. Morphometric Data of the Lesion, Psychophysical and Electrophysiological Parameters Observed in Myopic CNV Eyes

\begin{tabular}{|c|c|c|c|c|}
\hline \multirow[b]{2}{*}{ Parameter } & \multirow[b]{2}{*}{$\begin{array}{l}\text { Control Eyes } \\
\quad(n=16)\end{array}$} & \multicolumn{3}{|c|}{ Myopic CNV Eyes $(n=14)$} \\
\hline & & Baseline & 15 Days & 90 Days \\
\hline GDL $(\mu \mathrm{m})$ & $0.0 \pm 0.0$ & $\begin{array}{c}929.2 \pm 298.7 \\
\mathrm{~F}(1,28)=155.6, P<0.01\end{array}$ & $\begin{array}{c}582.5 \pm 194.6 \\
\mathrm{~F}(1,26)=13.2, P<0.01\end{array}$ & $\begin{array}{c}721.0 \pm 234.1 \\
\mathrm{~F}(1,26)=4.21, P=0.050\end{array}$ \\
\hline VA (logMAR) & $0.0 \pm 0.0$ & $\begin{array}{c}0.607 \pm 0.289 \\
\mathrm{~F}(1,28)=70.95, P<0.01\end{array}$ & $\begin{array}{c}0.364 \pm 0.115 \\
\mathrm{~F}(1,26)=8.54, P<0.01\end{array}$ & $\begin{array}{c}0.500 \pm 0.251 \\
\mathrm{~F}(1,26)=1.09, P=0.305\end{array}$ \\
\hline SLO-CM (dB) & $23.3 \pm 1.08$ & $\begin{array}{c}16.5 \pm 5.46 \\
\mathrm{~F}(1,28)=23.87, P<0.01\end{array}$ & $\begin{array}{c}21.58 \pm 4.95 \\
\mathrm{~F}(1,26)=6.65, P=0.01\end{array}$ & 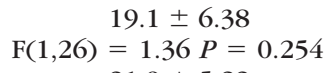 \\
\hline SLO-PM (dB) & $22.3 \pm 1.34$ & $\begin{array}{c}19.4 \pm 6.054 \\
\mathrm{~F}(1,28)=3.06, P=0.092\end{array}$ & $\begin{array}{c}22.8 \pm 4.81 \\
\mathrm{~F}(1,26)=2.71, P=0.112\end{array}$ & $\begin{array}{c}21.9 \pm 5.22 \\
\mathrm{~F}(1,26)=1.37, P=0.253\end{array}$ \\
\hline PERG 2P amplitude $(\mu \mathrm{V})$ & $1.26 \pm 0.123$ & $\begin{array}{c}0.586 \pm 0.129 \\
\mathrm{~F}(1,28)=214.2, P=0.01\end{array}$ & $\begin{array}{c}0.843 \pm 0.116 \\
\mathrm{~F}(1,26)=30.7, P<0.01\end{array}$ & $\begin{array}{c}0.750 \pm 0.145 \\
\mathrm{~F}(1,26)=10.9, P<0.01\end{array}$ \\
\hline PERG 2P phase (deg.) & $-97.3 \pm 2.31$ & $\begin{array}{c}-151.7 \pm 10.31 \\
\mathrm{~F}(1,28)=278.1, P<0.01\end{array}$ & $\begin{array}{c}-122.1 \pm 8.7 \\
\mathrm{~F}(1,26)=67.48, P<0.01\end{array}$ & $\begin{array}{c}-131.4 \pm 9.4 \\
\mathrm{~F}(1,26)=29.67, P<0.01\end{array}$ \\
\hline FERG 1F amplitude $(\mu \mathrm{V})$ & $1.36 \pm 0.146$ & $\begin{array}{c}0.521 \pm 0.167 \\
\mathrm{~F}(1,28)=215.6, P<0.01\end{array}$ & $\begin{array}{c}0.857 \pm 0.191 \\
\mathrm{~F}(1,26)=24.5, P<0.01\end{array}$ & $\begin{array}{c}0.729 \pm 0.144 \\
\mathrm{~F}(1,26)=12.4, P<0.01\end{array}$ \\
\hline FERG 1F phase (deg.) & $-98.3 \pm 2.72$ & $\begin{array}{c}-159.4 \pm 11.09 \\
\mathrm{~F}(1,28)=371.2, P<0.01\end{array}$ & $\begin{array}{c}-131.2 \pm 7.9 \\
\mathrm{~F}(1,26)=60.05, P<0.01\end{array}$ & $\begin{array}{l}-144.3 \pm 6.8 \\
\mathrm{~F}(1,26)=18.86, P<0.01\end{array}$ \\
\hline
\end{tabular}

Data were collected at baseline and 15 and 90 days after PDT. The data in CNV eyes at baseline were compared to data in control eyes, by ANOVA. The data observed in CNV eyes at 15 and 90 days after PDT were compared to baseline, by ANOVA. $\mathrm{N}=$ number of eyes enrolled. GDL $=$ greatest diameter of the lesion (choroidal neovascularization).

\section{Discussion}

In our study we assessed the effects of PDT on the macular function of myopic CNV eyes by evaluating psychophysical (VA, SLO microperimetry) and electrophysiological (FERG and PERG) responses before and 15 and 90 days after treatment with verteporfin.

At baseline (before treatment), myopic CNV eyes showed, compared with CEs, a significant decrease in VA, in central macular sensitivity tested by SLO microperimetry, and in PERG and FERG amplitudes. Paracentral macular sensitivity was reduced, though not significantly, when compared with CEs. This nonsignificant reduction could be ascribed to the variability of SLO-PM sensitivity in the group of CNV eyes and its correlation with the dimensions of the lesion. In fact, in eyes with a smaller lesion (prevalently affecting the central $2^{\circ}$ ), we found high SLO-PM sensitivity. In contrast, a concomitant reduction in SLO-PM sensitivity was observed in those patients with a large lesion (with an extension greater than the central $2^{\circ}$ ).

All parameters of visual dysfunction correlated significantly with the diameter of the lesion.

According to previous studies, ${ }^{14-17,31}$ the impairment of visual function and the loss of sensitivity observed in myopic CNV eyes may be related to the histologic changes occurring in the retinal structures. In fact, $\mathrm{CNV}$ in high myopia appears as a neovascular coinlike formation that develops under and through the RPE layer, strictly related to the presence of lac- quer cracks. These new vessels leak blood and fluid and cause a build-up of fibroblasts and neovascular endothelial cells between and within the RPE and photoreceptor layers, causing, in the early stages, a detachment of the RPE and retina. A persistent fibrovascular scar subsequently forms with a progressive loss of photoreceptors. ${ }^{31-34}$

The presence of concomitant abnormal FERG and PERG responses in $\mathrm{CNV}$ eyes may indicate that the functional impairment is present, not only in the outer macular layers (preganglionic elements), ${ }^{21-27}$ but also in the innermost macular layers (ganglion cells and their fibers). ${ }^{21,27-29}$ Whether the observed dysfunction of the innermost macular layers (abnormal PERG) should be considered a primary impairment caused by CNV or whether it is a consequence of the loss of photoreceptors has not been established. ${ }^{31-34}$

At 15 days after PDT, myopic CNV eyes showed a significant reduction in the diameter of the lesion related to the increase in VA and SLO-CM sensitivity, while SLO-PM sensitivity was substantially unmodified. In addition, we found a significant increase in PERG and FERG amplitudes, indicating an improvement in both the innermost (ganglion cells and their fibers) and outer (preganglionic elements) macular layers. ${ }^{21-29}$ Our findings are consistent with those of Moschos et al. ${ }^{35}$ who observed an increase in amplitude of multifocal (mf)ERG after PDT. However, mfERG was assessed 6 months after PDT. ${ }^{35}$

The improvement in visual function was significantly related to the reduction of the diameter of the lesion. This result

TABLE 3. Linear Regression and Correlations

\begin{tabular}{|c|c|c|c|c|c|}
\hline & $\begin{array}{c}\text { VA } \\
(\log \text { MAR) }\end{array}$ & $\begin{array}{l}\text { SLO-CM } \\
\text { (dB) }\end{array}$ & $\begin{array}{l}\text { SLO-PM } \\
\text { (dB) }\end{array}$ & $\begin{array}{c}\text { PERG 2P Amplitude } \\
(\log \mu V)\end{array}$ & $\begin{array}{c}\text { FERG 1F Amplitude } \\
\qquad(\log \mu \mathrm{V})\end{array}$ \\
\hline \multicolumn{6}{|c|}{ Diameter of lesion $(\log \mu \mathrm{m})$} \\
\hline$r$ & 0.882 & -0.727 & -0.672 & -0.801 & -0.843 \\
\hline$P$ & 0.00003 & 0.00321 & 0.0081 & 0.00057 & 0.00015 \\
\hline \multicolumn{6}{|c|}{ VA (logMAR) } \\
\hline$r$ & - & -0.794 & -0.798 & -0.701 & -0.637 \\
\hline$P$ & - & 0.00068 & 0.00062 & 0.00522 & 0.010004 \\
\hline
\end{tabular}

Correlations were determined between the diameter of the lesion and parameters of visual function and between VAs and the other parameters of visual function observed in CNV eyes at baseline. 


\section{CNV eyes: 15 days after PDT}

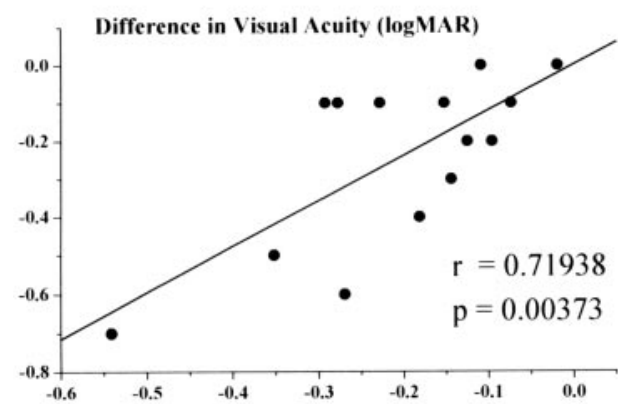

12 Difference in SLO-CM (dB)
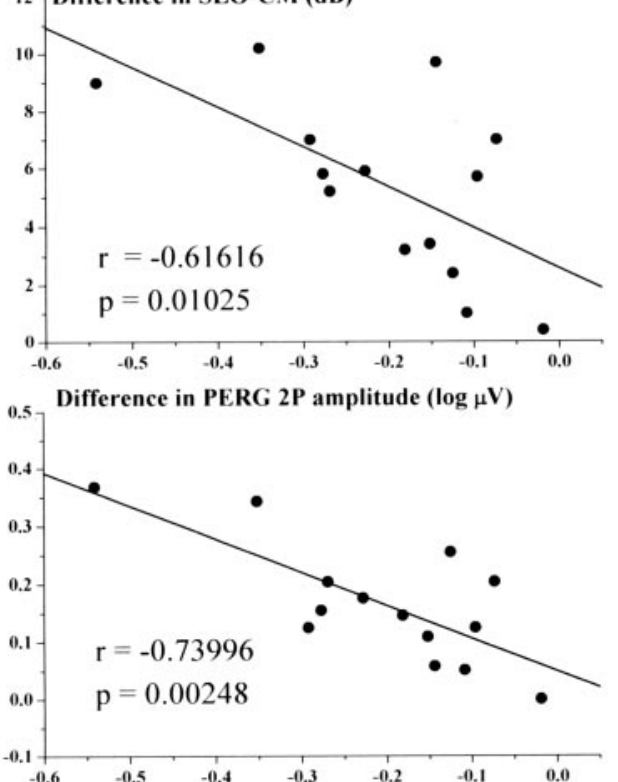

Difference in F-ERG IF amplitude $(\log \mu \mathrm{V})$

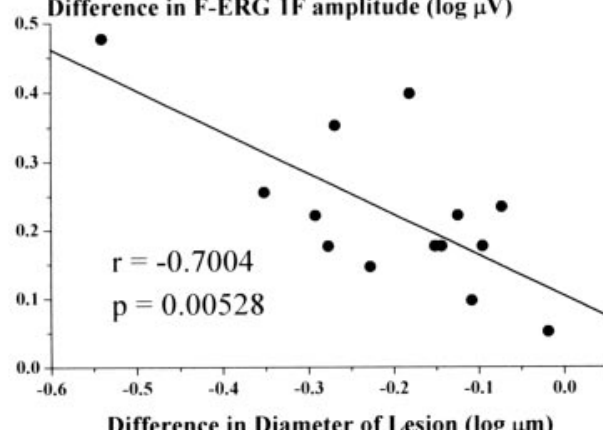

Difference in Diameter of Lesion $(\log \mu \mathrm{m})$

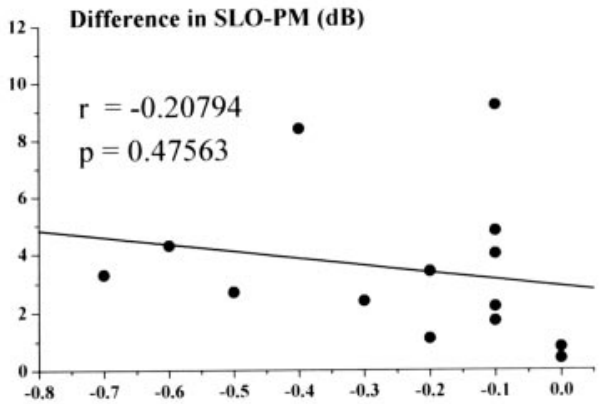

12. Difference in SLO-CM (dB)

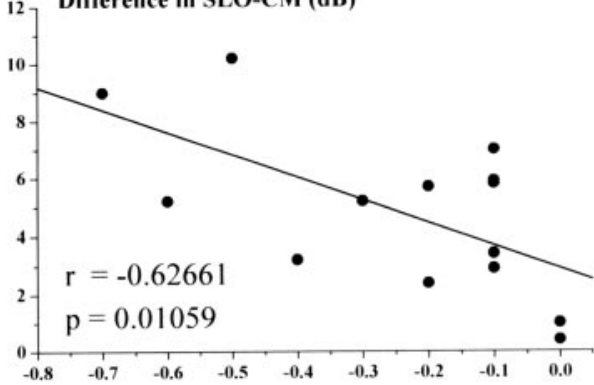

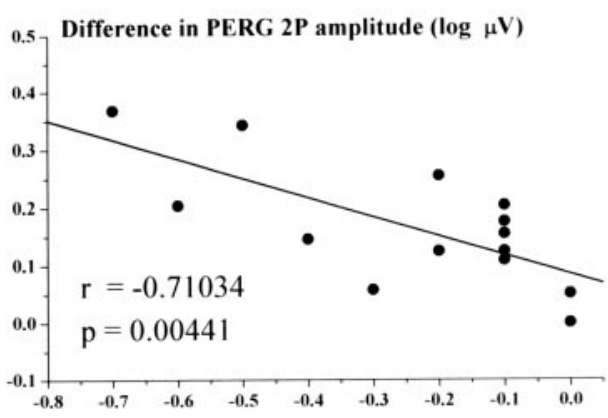

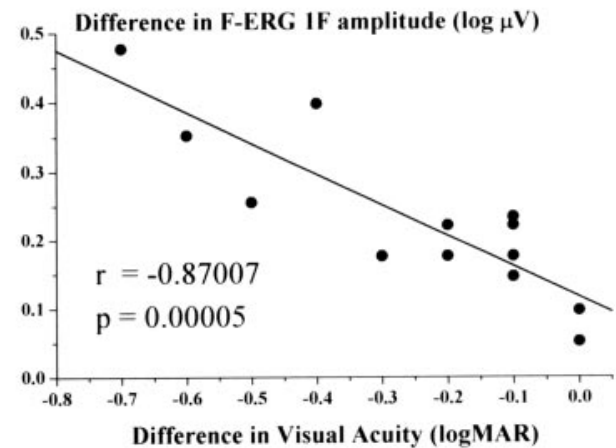

Figure 4. Left: differences in the diameter of the lesion observed in myopic CNV eyes 15 days after PDT in relation to baseline, plotted against the differences in logMAR VA, SLO-CM sensitivity, and PERG 2P and FERG 1F amplitudes. Right: differences in logMAR VA in myopic CNV eyes 15 days after PDT in relation to baseline, plotted against the differences in SLO-CM and -PM sensitivities and PERG 2P and FERG $1 \mathrm{~F}$ amplitudes. The Pearson test was used for regression analysis.

is in line with the reported effects of PDT on choroidal and retinal structures. Indeed, PDT exerts noxious effects on vascular endothelial cells of the choriocapillary layer, with the consequent obliteration of $\mathrm{CNV},{ }^{36}$ whereas the neurosensory retina, including photoreceptors and retinal capillaries, is preserved in PDT-treated areas. ${ }^{36}$

Several recent studies provide possible explanations regarding the noxious effects of PDT on choriocapillary endothelial cells. ${ }^{36-40}$ The damage may be due to a cytotoxic mechanism caused by the generation of single oxygen. This reactive substance interacts with the lipids of the cytoplasmic membrane and with other intracellular components, causing shrinkage of endothelial cells and exposure of the basal membrane. These events lead to an intensive thrombogenic stimulus: activated platelets release vasoactive mediators causing stasis of blood flow due to vasoconstriction, platelet adhesion, erythrocyte sludging, and increased vascular permeability. Finally, the complete occlusion of capillary lumina by fibrin, platelets, and cellular debris occurs. Recanalization of physiological choroids is precociously observed 1 week after PDT. ${ }^{36-40}$

The high selectivity of verteporfin for neovascular structures compared with normal choroidal vasculature has been ascertained. In fact, verteporfin binds to endogenous lipoproteins (LDLs), which are overexpressed in neovascular endothelial cells, leading to occlusion of the neovascular membrane and restoration of the vascular barrier function, reducing extravascular fluid and hemorrhage and providing a reattachment of RPE and photoreceptors. ${ }^{38,40}$ Histologic studies have revealed the structural integrity of photoreceptors and Bruch's membrane after PDT, perhaps due to the maintained perfusion of the deeper choroidal vasculature. ${ }^{36}$ 


\section{CNV eyes: 90 days after PDT}
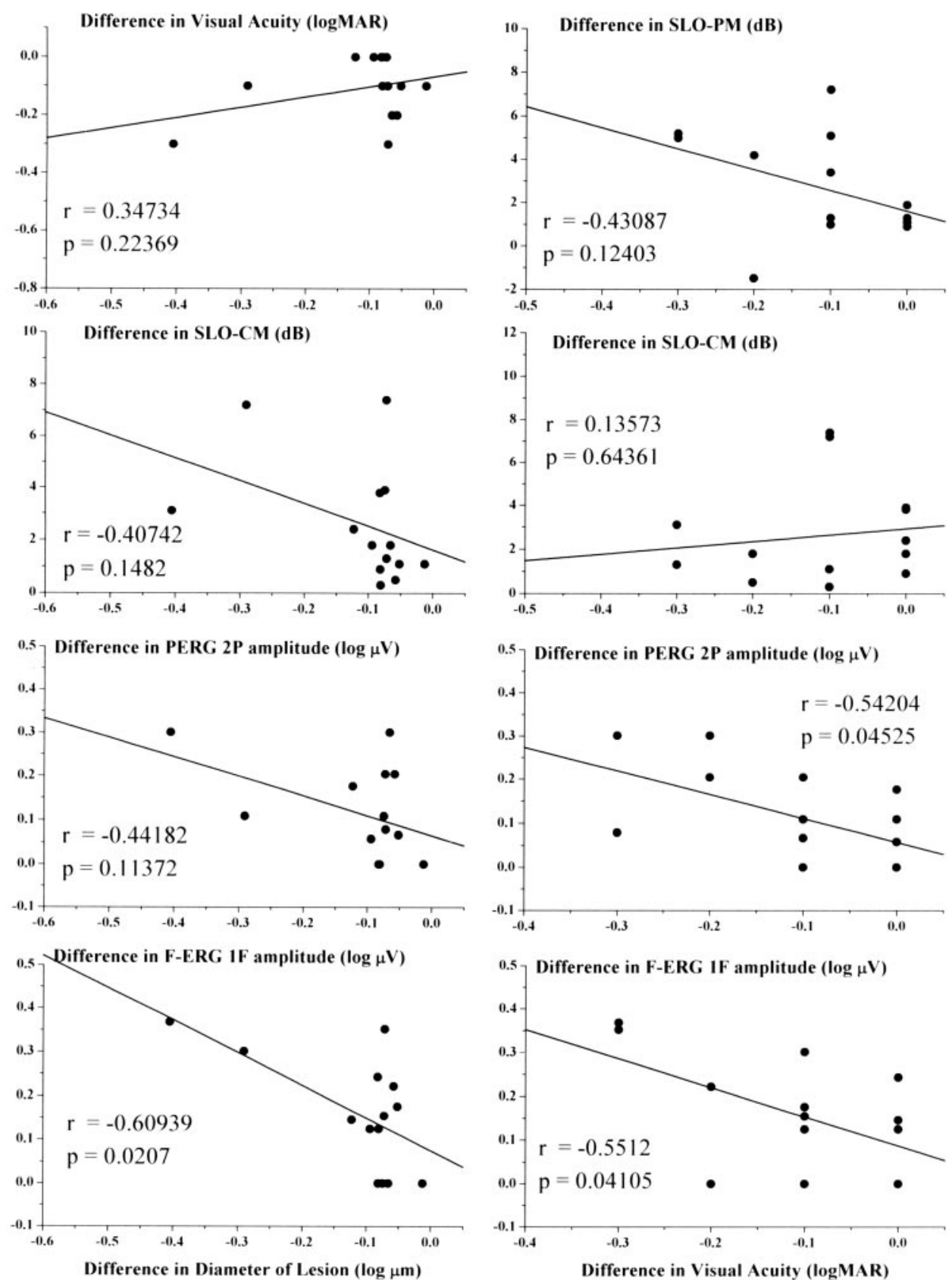

Figure 5. Differences in (left) the diameter of the lesion and (right) logMAR VA observed in myopic CNV eyes 90 days after PDT. The remainder of the description is as in Figure 4 , but is for the 90-day follow-up examination and measurements.
All this could explain the improvement of visual function (VA, SLO-CM, FERG, and PERG) after treatment, as well as the angiographic features (decrease in the greatest linear diameter of the lesion) in the early stages of treatment. In fact, 15 days after PDT, fluorescein angiography of CNV eyes revealed an absence of leakage in 13 of the 16 eyes, and in the other 3 eyes there was a great reduction in leakage. This is in agreement with other studies that documented hypoperfusion of the choriocapillary layer together with a transient closure of $\mathrm{CNV}$ during the first week. 38,39

The results of the sensitive tests of photoreceptor function (SLO-CM and FERG) suggest that the transient choriocapillary occlusion reported is compatible, not only with the maintenance of visual function but also with the improvement of retinal sensitivity within the treated $\mathrm{CNV}$ area. Regarding the observed functional improvement of the innermost macular layers (an increase in amplitudes and a shortening in phases of PERG recordings), it is not possible to establish whether it is a primary effect of PDT on ganglion cells and their fibers or it is the consequence of the improvement of photoreceptor function.

At 90 days after PDT, myopic CNV eyes show an increase in the diameter of the lesion and a worsening of VA, SLO-CM sensitivity, and FERG and PERG amplitudes and phases in relation to the values observed after 15 days. Nevertheless, all parameters of visual function were higher (VA and SLO-CM not significantly, but PERG and FERG amplitudes and phases still significantly) in relation to baseline. Our VA results are consistent with those of Montero et al. ${ }^{17}$ and with those of Parodi et al. ${ }^{31}$ who found, at 3 months after PDT, VA was similar to that observed before PDT. 
The presence at 90 days of a substantially unmodified VA with a concomitant functional improvement of the outer and inner macular layers (FERG and PERG with increased amplitude and shortened phases) in relation to baseline values could appear a contradictory result. However, it is possible that the functional improvement assessed by electrophysiological methods is not sufficient to determine a significant improvement in psychophysical responses (VA and SLO-CM).

This subsequent worsening of visual function could in part be explained by the morphologic changes of the area of the lesion. In fact, fluorescein angiography revealed an increase in the diameter of the lesion 90 days after PDT when compared with the assessment 15 days after PDT and, in addition, a reappearance of leakage in 5 of the 14 myopic $\mathrm{CNV}$ eyes. Similar to our results, several studies reported a relapse of the lesion in the CNV area or the absence of significant changes in relation to pretreatment findings,${ }^{17,31}$ whereas in the Verteporfin in Photodynamic Therapy (VIP) study, fluorescein angiography was performed at 12 and 24 months after PDT, and thus it is not comparable to our study. ${ }^{14}$

The lack of correlation between changes in the diameter of the lesion and changes in VA, SLO-CM sensitivity, and FERG and PERG amplitudes could suggest that the visual function observed after PDT is not solely related to the dimension of lesion. Other events occurring in the area of the lesion may play a role, such as fluorescein leakage, breakdown of the outer blood-retinal barrier (responsible for macular edema), and the presence of blood.

Our results regarding changes in the area of myopic CNV (diameter of the lesion, leakage, intralesional blood) are in agreement with those in other studies ${ }^{14,17,31}$ suggesting that PDT may not only prevent the progression of CNV but, in some cases, may also induce a regression of $\mathrm{CNV}$ in relation to pretreatment status.

In conclusion, in this open study, performed in a selected series of patients, we observed that 15 days after treatment in myopic CNV eyes, PDT induced a reduction in the diameter of the lesion together with an improvement of the function of both ganglionic and preganglionic elements of the macular region (as suggested by the improvement in FERG and PERG responses), leading to increased VA and macular sensitivity, as assessed by SLO-CM. Ninety days after PDT, we observed a worsening of the CNV lesion with consequent reduced function of both ganglionic and preganglionic elements and related decline of VA and SLO-CM sensitivity that were substantially unmodified in relation to pretreatment results.

Thus, our results indicate that, in CNV eyes after PDT, there is an initial improvement followed by a progressive decline of macular function until a state similar to baseline is obtained. Nevertheless, considering that at the same time of follow-up (90 days) untreated CNV eyes showed a worsening trend in visual impairment, ${ }^{14,16}$ our findings could suggest the potential use of PDT to obtain, though short-term (90 days), the reduction of the progression of macular dysfunction.

\section{References}

1. Tabandeh HT, Flynn HW, Scott IU, et al. VA outcomes of patients 50 years of age and older with high myopia and untreated choroidal neovascularization. Ophthalmology. 1999;106:2063-2067.

2. Fleury I, De Laey JJ. Prognosis of the disciform response in high myopia. Bull Soc Belge Ophthalmol. 1983;206:91-102.

3. Secrétan M, Kuhn D, Soubrane G, Coscas GJ. Long-term visual outcome of choroidal neovascularization in pathologic myopia: natural history and laser treatment. Eur J Ophthalmol. 1997;7: 307-316.

4. Cohen SY, Laroche A, Leguen Y, Soubrane G, Coscas GJ. Etiology of choroidal neovascularization in young patients. Ophthalmology. 1996;103:1241-12444.
5. Hampton RG, Kohen D, Bird AC. Visual prognosis of disciform degeneration in myopia. Ophthalmology. 1983;90:923-926.

6. Hotchkiss ML, Fine SL. Pathologic myopia and choroidal neovascularization. Am J Ophthalmol. 1981;91:177-183.

7. Soubrane G, Pisan J, Bornert P, Perrenoud F, Coscas G. Néovaisseaux sous-rétiniens de la myopie dégénérative: rèsultats de la photocoagulation. Bull Soc Ophtalmol Fr. 1986;86:269-272.

8. Deutman AF, Hendikse F, Hoyng C. Traitement des néovaisseaux sous-rétiniens dans la myopie dégénérative. Ophthalmologie. 1989;3:299-301.

9. Thomas MA, Dickinson JD, Melberg NS, et al. Visual results after surgical removal of subfoveal choroidal neovascular membranes. Ophthalmology. 1994;101:1384-1396.

10. Adelberg DA, Del Priore LV, Kaplan HJ. Surgery for subfoveal membranes in myopia, angioid streaks, and other disorders. Retina. 1995;15:198-205.

11. TAP Study Group. Photodynamic therapy of subfoveal choroidal neovascularization in age-related macular degeneration with verteporfin: one-year results of 2 randomized clinical trials. TAP Report 1. Arch Ophthalmol. 1999;117:1329-1345.

12. TAP Study Group. Photodynamic Therapy of Subfoveal Choroidal Neovascularization in Age-Related Macular Degeneration with Verteporfin: two-year results of 2 randomized clinical trials. TAP report 2. Arch Ophthalmol. 2001;119:198-207.

13. Blinder KJ, Bradley S, Bressler NM, et al. Effect on lesion size, VA, and lesion composition on VA change with and without verteporfin therapy for choroidal neovascularization secondary to agerelated macular degeneration: TAP and VIP report no 1 . Am J Ophthalmol. 2003;136:407-418.

14. VIP Study Group. Photodynamic Therapy of Subfoveal Choroidal Neovascularization in Pathologic Myopia with Verteporfin: oneyear results of a randomised clinical trials. VIP report 1. Ophthalmology. 2001;108:841-852.

15. TAP and VIP Study Groups. Photodynamic therapy Of Subfoveal Choroidal Neovascularization with Verteporfin: fluorescein angiographic guidelines for evaluation and treatment. TAP and VIP report 2. Arch Ophthalmol. 2003;121:1253-1268.

16. VIP Study Group. Verteporfin Therapy of Subfoveal Choroidal Neovascularization in Pathologic Myopia: 2-year results of a randomized clinical trial. VIP report No 3. Ophthalmology. 2003;110: 667-673.

17. Montero JA, Ruiz-Moreno JM. Verteporfin photodynamic therapy in highly myopic subfoveal choroidal neovascularization. $\mathrm{Br} \mathrm{J}$ Ophthalmol. 2003;87:173-176.

18. Varano M, Scassa C. Scanning laser ophthalmoscope microperimetry. Semin Ophthalmol. 1998;13:203-209.

19. Rohrschneider K, Fendrich T, Becker M, et al. Static fundus perimetry using the scanning laser ophthalmoscope with an automated threshold strategy. Graefes Arch Clin Exp Ophthalmol. 1995;233:743-749.

20. Rohrschneider K, Becker M, Schumacher N, et al. Normal values for fundus perimetry with the scanning laser ophthalmoscope. Am J Ophthalmol. 1998;126:52-58.

21. Parisi V, Falsini B. Electrophysiological evaluation of the macular cone system: focal electroretinography and visual evoked potentials after photostress. Semin Ophthalmol. 1998;13:178-188.

22. Seiple WH, Siegel IM, Carr RE, Mayron C. Evaluating macular function using the focal ERG. Invest Ophthalmol Vis Sci. 1986;27: 1123-1130

23. Falsini B, Fadda A, Iarossi G, et al. Retinal sensitivity to flicker modulation: reduced by early age-related maculopathy. Invest Ophthalmol Vis Sci. 2000;41:1498-1506.

24. Miyake Y, Shiroyama N, Ota I, Horiguchi M. Local macular electroretinographic responses in idiopathic central serous chorioretinopathy. Am J Ophthalmol. 1988;106:546-550.

25. Iarossi G, Falsini B, Piccardi M. Regional cone dysfunction in retinitis pigmentosa evaluated by flicker ERGs: relationship with perimetric sensitivity losses. Invest Ophthalmol Vis Sci. 2003;44: 866-874.

26. Parisi V, Canu D, Iarossi G, Olzi D, Falsini B. Altered recovery of macular function after bleaching in Stargardt's disease-fundus flavimaculatus: pattern VEP evidence. Invest Ophthalmol Vis Sci. 2002; $43: 2741-2748$ 
27. Porciatti V, Falsini B, Fadda A, Bolzani R. Steady-state analysis of the focal ERG to pattern and flicker: relationship between ERG components and retinal pathology. Clin Vis Sci. 1989;4:323-332.

28. Falsini B, Bardocci A, Porciatti V, Bolzani R, Piccardi M. Macular dysfunction in multiple sclerosis revealed by steady-state flicker and pattern ERGs. Electroencephalogr Clin Neurophysiol. 1992; 82:53-59.

29. Parisi V, Pierelli F, Restuccia R, et al. VEP after photostress response in multiple sclerosis patients previously affected by optic neuritis. Electroencephalogr Clin Neurophysiol. 1998;108:73-79.

30. Fiorentini A, Maffei L, Pirchio M, et al. The ERG in response to alternating gratings in patients with diseases of the peripheral visual pathway. Invest Opbthalmol Vis Sci. 1981;21:490-493.

31. Parodi MB, Da Pozzo S, Ravalico G. Angiographic features after photodynamic therapy for choroidal neovascularisation in age related macular degeneration and pathological myopia. $\mathrm{Br} \mathrm{J} \mathrm{Oph-}$ thalmol. 2003;87:177-183.

32. Grossniklaus HE, Gass JDM. Clinicopathologic correlations of surgically excised type 1 and type 2 submacular choroidal neovascular membranes. Am J Opbthalmol. 1998;126:59-69.

33. Coscas $G$, Soubrane $G$. Choroidal neovascularisation in degenerativemyopia. In: Ryan SJ, ed. Retina (Italian version). Vol. 2. St. Louis: CV Mosby Co.; 1992;:211-222.
34. Avila MP, Weiter JJ, Jalkh AE, Trempe CL, Pruett RC, Schepens CL. Natural history of choroidal neovascularization in degenerative myopia. Ophthalmology. 1984;91:1573-1581.

35. Moschos MN, Panayotidis D, Moschos MM, et al. A preliminary assessment of macular function by MFERG in myopic eyes with CNV with complete response to photodynamic therapy. Eur J Opbthalmol. 2003;13:461-467.

36. Schmidt-Erfuth U, Laqua H, Schlotzer-Schrehardt U, et al. Histopathological changes following photodynamic therapy in human eyes. Arch Ophthalmol. 2002;120:835-844.

37. Schmidt-Erfurth U, Hasan T. Mechanism of action of photodynamic therapy with verteporfin for the treatment of age-related macular degeneration. Surv Ophthalmol. 2000;45:195-214.

38. Michels S, Schmidt-Erfurth U. Sequence of early vascular events after photodynamic therapy. Invest Ophthalmol Vis Sci. 2003;144: 2147-2154.

39. Schmidt-Erfurth U, Michels S, Barbazzetto I, et al. Photodynamic effects on choroidal neovascularization and physiological choroid. Invest Ophthalmol Vis Sci. 2002;43:830-841.

40. Schlotzer-Schrehardt U, Viestenz A, Naumann GO, et al. Dose related structural effects of photodynamic therapy oh choroidal and retinal structures of human eyes. Graefes Arch Clin Exp Opbthalmol. 2002;240:748-757. 